Wire-like characteristics in stacked InAs/GaAs quantum dot superlattices for optoelectronic devices

This article has been downloaded from IOPscience. Please scroll down to see the full text article.

2007 Semicond. Sci. Technol. 221077

(http://iopscience.iop.org/0268-1242/22/9/017)

The Table of Contents and more related content is available

Download details:

IP Address: 140.112.113.225

The article was downloaded on 26/06/2009 at 11:10

Please note that terms and conditions apply. 


\title{
Wire-like characteristics in stacked InAs/GaAs quantum dot superlattices for optoelectronic devices
}

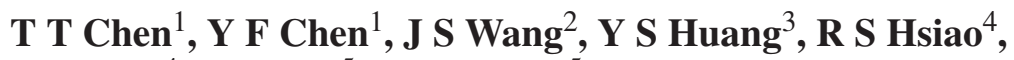 \\ J F Chen ${ }^{4}$, C M Lai ${ }^{5}$ and J Y Chi ${ }^{5}$ \\ ${ }^{1}$ Department of Physics, National Taiwan University, Taipei 106, Taiwan \\ 2 Department of Physics, Chung Yuan Christian University, Chung-Li 32023, Taiwan \\ ${ }^{3}$ Department of Electronic Engineering, National Taiwan University of Science and \\ Technology, Taipei 106, Taiwan \\ ${ }^{4}$ Department of Eletrophysics, National Chiao Tung University, Hsinchu 300, Taiwan \\ ${ }^{5}$ Industrial Technology Research Institute (OES/ITRI), Hsinchu 310, Taiwan \\ E-mail: yfchen@phys.ntu.edu.tw
}

Received 15 May 2007, in final form 24 July 2007

Published 23 August 2007

Online at stacks.iop.org/SST/22/1077

\begin{abstract}
The wire-like characteristics of stacked InAs/GaAs quantum dot (QDs) superlattices induced by the vertically electronic coupling effect were demonstrated by surface photovoltaic and photoluminescence measurements. It was found that the surface photovoltaic signal can be enhanced by up to more than 100 times due to the wire-like behavior along the growth direction. We also found that the emission from the cleaved edge surface is strongly anisotropic, which suggests a possibility of fine tuning the polarization by changing the spacer thickness. Additionally, the electroluminescence of stacked QDs near $1.3 \mu \mathrm{m}$ based on the wire-like characteristics has a much better performance than that of uncoupled QDs.
\end{abstract}

\section{Introduction}

Self-assembled semiconductor quantum dots (QDs) have attracted much attention due to the realistic prospect of improved optical and electronic properties with the potential benefits of zero-dimensional characteristics [1]. In terms of applications, one of the important aspects of QDs is vertical stacking of self-assembled QDs along the growth direction, which results from the strain field interaction during the growth process [1-3]. The vertically stacked InAs/GaAs QDs can largely modify the structural and electronic properties due to strain variation [4], intermixing of the constituent atoms [5] and electronic coupling between the QDs [6, 7]. In contrast to the single QD layer, the large QD density in the QD superlattices is able to increase the active volume and enhance the modal gain in a light-emitting device [1,8]. Compared with an individual QD, the vertically stacked QDs are expected to exhibit quasi-one-dimensional wirelike characteristics induced by vertically electronic coupling. In order to have a better use of the unique properties of stacked QDs in a practical optoelectronic device, a complete understanding of the effects generated by the electronic coupling is required. In this paper, we show that the surface photovoltage (SPV) signal can be enhanced by up to more than 100 times due to the vertically electronic coupling effect. The emission from the cleaved edge surface is strongly anisotropic, which provides an opportunity to fine tune the polarization by changing spacer thickness. Furthermore, we show that the electroluminescence (EL) near $1.3 \mu \mathrm{m}$ based on the wire-like behavior of stacked QDs has great potential applications in a single mode QD laser.

\section{Experiment}

The studied InAs/GaAs QD superlattices were grown on $\mathrm{n}^{+}$GaAs ( 1000$)$ substrates via the Stranski-Krastanow (SK) growth mode by solid source molecular beam epitaxy (SSMBE) in a Riber-Epineat machine [1]. After an undoped GaAs buffer layer, the sample consists of a $30 \mathrm{~nm}$ thick AlAs bottom cladding layer, 30 stacks of self-assembled InAs QD layers with $30 \mathrm{~nm}(20 \mathrm{~nm}, 16 \mathrm{~nm}$ and $10 \mathrm{~nm})$ thick GaAs 


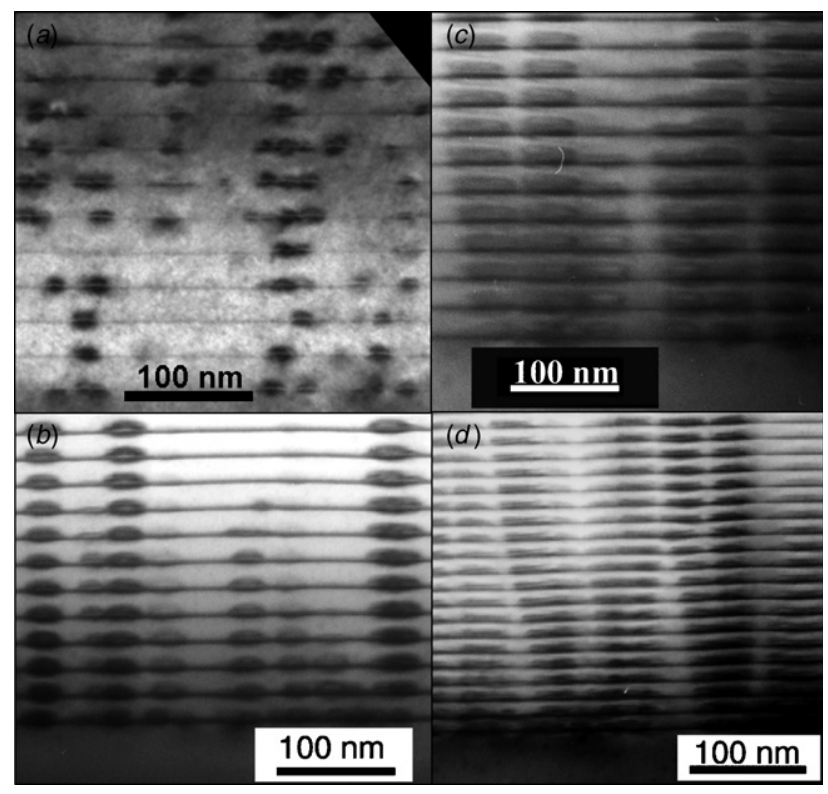

Figure 1. Cross-sectional transmission electron microscopy images of the samples growth with different GaAs spacer layer thickness.

spacer layers, a $30 \mathrm{~nm}$ thick AlAs top cladding layer and a $5 \mathrm{~nm}$ thick GaAs cap layer. The QDs were obtained by the deposition of InAs 2.6 monolayers (MLs) with a substrate temperature of $485^{\circ} \mathrm{C}$, and then by covering these layers with a $10 \mathrm{~nm}$ thick GaAs layer at the same temperature without growth interruption. The growth temperature of the remaining layers was $600^{\circ} \mathrm{C}$. The formation of QDs was controlled in situ by monitoring the diffraction pattern of high-energy electrons (RHEED). In addition, a sample containing a single layer of InAs QDs (2.6 MLs) was used for comparison.

Transmission electron microscope (TEM) images were performed using a Philips Tecnai-20 instrument operating at $200 \mathrm{keV}$. For the photoluminescence (PL) measurement, the signal was recorded by a $0.3 \mathrm{~m}$ single-grating spectrometer and an InGaAs detector. An Ar-ion laser working at $514.5 \mathrm{~nm}$ was used as the excitation source. For the polarizationdependent measurement of the PL spectra from the cleaved edge surface, a depolarizing filter was placed in front of the spectrometer to exclude the possible polarized characteristic of the grating. For the SPV measurement, the contact potential difference between the sample and a reference grid electrode was measured in a capacitive manner as a function of the photon energy of the incident light. The SPV measurement provided information about those electrons and holes created by the incident light with different wavelengths, which did not recombine radiatively. Thus, the SPV adsorption spectrum was being used as a complementary method to the PL emission spectrum.

\section{Results and discussion}

Figure 1 shows the [ 1000$]$-axial cross-sectional TEM images of 30 periodic InAs QDs growth with (a) $30 \mathrm{~nm}$, (b) $20 \mathrm{~nm}$, (c) $16 \mathrm{~nm}$ and (d) $10 \mathrm{~nm}$ GaAs spacer layer thickness. The vertical alignment of QDs is due to the strain field of buried QDs in the underlying layer; thus the vertical pairing

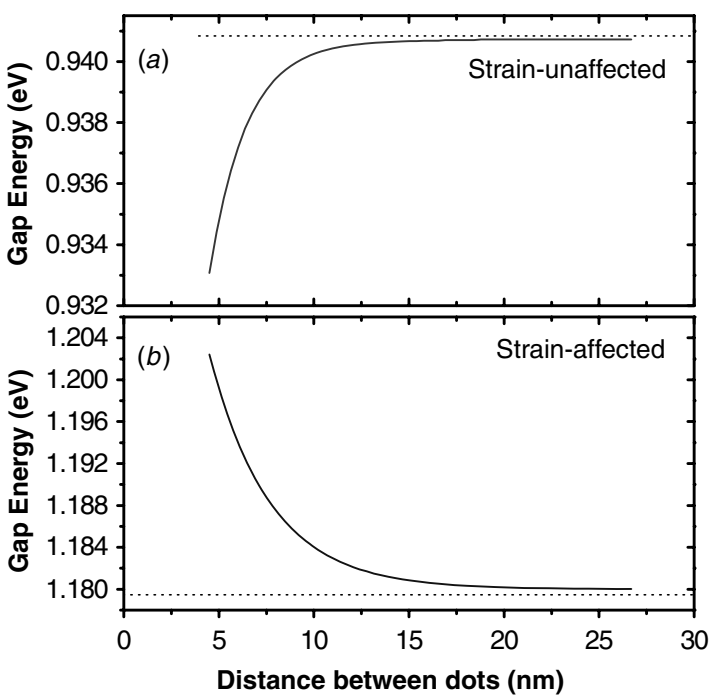

Figure 2. Band gap energy as a function of the distance between stacked quantum dots. The solid curves are exponential fits, and the horizontal dot lines are their asymptotes corresponding to the isolated quantum dot energy.

probability can be varied by the spacer thickness [2]. It is found that with decreasing spacer thickness, the vertical alignment of InAs QDs becomes better. As shown in figure 1(a), there exist isolated QDs and several vertically stacked QDs in the $30 \mathrm{~nm}$ spacer sample. For the $20 \mathrm{~nm}$ spacer sample, we can see a better vertical alignment of the QDs, and the size of the QDs increases with increasing number of stacking layers, which has also been attributed to the accumulation of strain field in the underlying QD layer [5]. As the spacer thickness decreases to $10 \mathrm{~nm}$, we can actually see that a complete vertical alignment of QDs is obtained as shown in figure 1(d) [4]. Additionally, we can see that the size of the QDs changes gradually with decreasing spacer thickness; however, the actual size of the QDs could be smaller since the strain field may extend beyond the QD boundary [4].

To begin with the discussion of our main results on the wire-like behaviors in stacked QDs, let us present the effect of electronic coupling on the energy levels as predicted by a theoretical calculation performed previously [6]. The influence of strain and electronic coupling on the electronic structure is considered within the $\mathrm{sp}^{3} \mathrm{~s}^{*}$ empirical tight binding (ETB) model with interactions up to the second nearest neighbors and spin-orbit coupling. Figure 2 shows the band gap energy as a function of the distance between vertically stacked QDs with and without strain effect adapted from the previous report [6]. As shown in figure 2(a), the band gap energy starts to decrease as the distance between stacked QDs becomes smaller than about $12 \mathrm{~nm}$, due to the effect of the vertically electronic coupling. However, as we can see in figure $2(b)$, the strain field dominates the energy level shifts, leading to opposite events as for electronic coupling. Previously, similar studies have also been performed by the eight-band strain-dependent $\mathbf{k} \cdot \mathbf{p}$ Hamiltonian [9]. It is found that the variation of the band gap energy induced by the vertically electronic coupling and strain effect is comparable with the results as shown in figure 2. Thus, for closely stacked QDs, the strain field induces vertical alignment of QDs during 


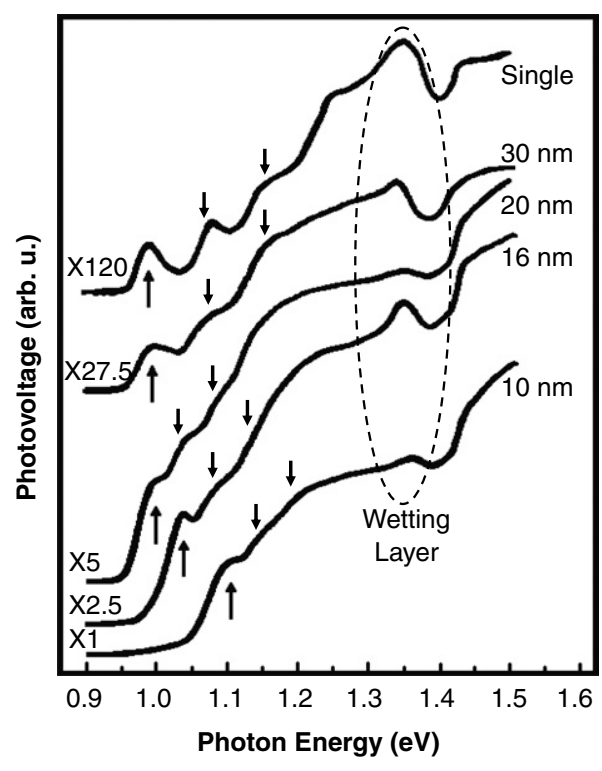

Figure 3. Surface photovoltaic spectra recorded at room temperature; the up-arrows and down-arrows indicate the ground state and the excited state transitions for each sample, respectively.

the growth process at first, and then the electronic coupling forms minibands and stimulates wire-like characteristics. Besides, the energy level shift may also be affected by the varied size of QDs in samples with different spacer thicknesses. As shown before [10], the energy level shift depends strongly on the base length of QDs, but is insensitive to the height and the volume of QDs [10]. According to figure 1, the base length of QDs increases slightly with decreasing spacer thickness. Similar to the vertically electronic coupling effect, the increase of the base length also causes the shrinkage of the band gap energy. However, it should be stressed here that even though the band gap energy is affected by the strain and the size effect, the effect of the electronic coupling still exists in the QD superlattices.

To demonstrate the wire-like behavior due to the vertically electronic coupling in QD superlattices, we have performed the SPV measurement which monitors the change in surface photovoltage caused by the incident photon. The SPV signal arises from the photoexcited carriers, which are spatially separated to the top and bottom sides of the sample under an electric field formed at the surface [11, 12]. As shown in figure 3, we can clearly see that the SPV signal increases when the spacer thickness is smaller than $20 \mathrm{~nm}$. Based on the theoretical calculation as shown in figure 2, the enhanced SPV signal with decreasing spacer thickness implies the formation of minibands, which enable the photoexcited carriers to travel easily along the vertical direction. With further decrease in the spacer thickness, the strength of the vertically electronic coupling increases. Thus, the photoexcited carriers can travel more freely along the vertical direction, the photoexcited electron-hole pairs will be more easily driven in the opposite direction, and the SPV signal will become larger. It is worth noting that the SPV signal can be enhanced by up to more than 100 times compared with that of a single InAs QD layer. We point out here that the giant enhancement of the SPV signal implies an enhanced absorption in stacked QDs
(100) 90

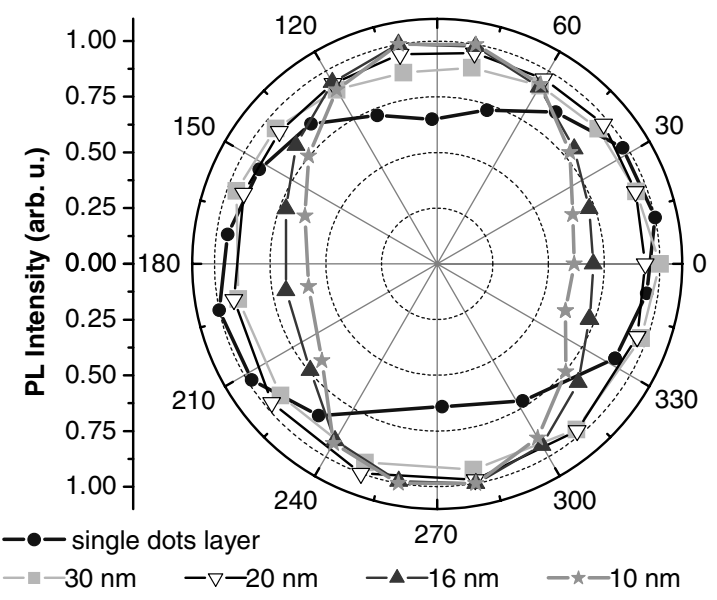

Figure 4. Polar plots of the polarized PL peak intensity (normalized).

which suggests a potential application of QD supperlattices in solar cell devices. Additionally, the energy separation of the ground and excited states transitions is almost the same for the single-layer QDs and the $30 \mathrm{~nm}$ sample as shown in figure 3. It implies that the electronic coupling does not play a significant role for the sample with a spacer thickness of $30 \mathrm{~nm}$, and therefore the corresponding SPV signal is comparable with that of the single QD layer. According to Gaussian fitting (not show here), the energy differences between the ground state and the first excited state are $90 \mathrm{meV}, 60 \mathrm{meV}$ and $54 \mathrm{meV}$ for the $30 \mathrm{~nm}, 16 \mathrm{~nm}$ and $10 \mathrm{~nm}$ samples, respectively. Apparently, the separation of the peak energy decreases with decreasing spacer thickness, yet another piece of evidence for the increased strength of the electronic coupling as shown in figure 2(a). Moreover, because of the level broadening due to the electronic coupling, the broadening of the SPV spectra is expected as the spacer thickness decreases as shown in figure 3.

Another important property to reveal the tunable wirelike characteristic due to different degree of the electronic coupling is the optical anisotropy of the edge emission. It arises from the dependence of the polarization of the emission on the confinement direction of the carriers involved. For the InAs/GaAs QDs, it is well known that the emission of the QDs should be polarized along the elongated direction of the electronic states $[13,14]$; therefore, we can use this property to verify the wire-like characteristics and the formation of minibands. It is worth noting that the change in the optical anisotropy as studied in previous reports is due to the shape of individual QDs in a single layer [13, 14], while in this paper the change in the optical anisotropy is caused by the vertically electronic coupling effect between different layers. Conventionally, the polarization degree is defined as $P=\left|\left(I_{\|}-I_{\perp}\right)\right| /\left(I_{\|}+I_{\perp}\right)$, where $I_{\|}\left(I_{\perp}\right)$ is the PL intensity polarized in (normal to) the surface plane. As shown in figure 4, the single-layer QDs and the sample with $30 \mathrm{~nm}$ spacer thickness both reveal a TE polarization (perpendicular to the growth direction) emission corresponding to the in-plane enlarged QD shape. As the spacer thickness decreases to $20 \mathrm{~nm}$, the edge emission is almost unpolarized, a fact that can 


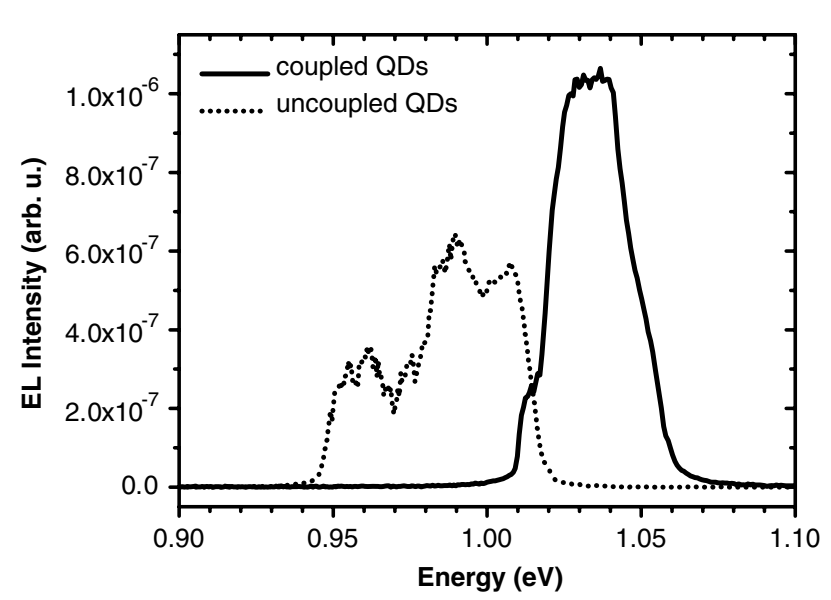

Figure 5. Electroluminescence spectra of vertically electronic coupled quantum dots (solid line), and the dashed line shows a similar uncoupled quantum dots.

be attributed to the enhanced vertically electronic coupling, which will induce TM polarization (parallel to the growth direction) and compensate for the initial TE polarization. By further decreasing the spacer thickness, we can actually see that the polarization turns into the TM behavior. For the $16 \mathrm{~nm}$ and $10 \mathrm{~nm}$ spacer samples, the degree of the TM polarization $P$ is $18 \%$ and $26 \%$, respectively. It is clear that the degree of the TM polarization increases with increasing strength of the vertically electronic coupling, and hence the wire-like behavior along the coupling direction is more pronounced.

To illustrate important consequences of the wire-like behavior of vertically stacked QDs, let us examine the EL measurements as shown in figure 5. The EL spectrum was recorded with an injection current of $1 \mathrm{~A}$ under $\mathrm{CW}$ operation. The sample of vertically electronic coupled QDs contains ten stacks of 2.6 MLs InAs QDs with $16 \mathrm{~nm}$ spacer thickness, and a similar sample with conventional uncoupled InAs/InGaAs/GaAs QDs (2.4 MLs InAs QD; $30 \mathrm{~nm}$ spacer thickness) was used for comparison. It is found that the line width of the emission from vertically electronic coupled QDs is about $29 \mathrm{meV}$, which is almost half of that obtained in uncoupled QDs. This behavior can be explained by the fact that in the uncoupled case, the photoexcited carriers can occupy both the excited and ground states of an individual QD at high injection current. However, for the vertically electronic coupled QDs with wire-like characteristics, the photoexcited carriers can tunnel and only accumulate at the ground state [1]. Therefore, the narrow line width of the EL in the coupled QDs is again a consequence of the electronic coupling. This result indicates that vertically electronic coupled QD superlattices have great potential for single-mode laser applications.

\section{Conclusion}

In conclusion, the interesting wire-like characteristics of InAs QDs due to the vertically electronic coupling have been clearly demonstrated. It is found that with decreasing spacer thickness, at first the strain field induces vertically aligned QDs, and then the wavefunction overlap stimulates wire-like characteristics. When the spacer thickness is smaller than the critical value, the SPV signal can be enhanced by up to 100 times, which provides an excellent opportunity for solarcell application. The edge-emitted light exhibits a tunable polarization along the growth direction by adjusting the spacer thickness. It is shown that the wire-like characteristics of vertically stacked QDs are suitable for the application of a high performance single-mode QD laser.

\section{Acknowledgments}

The authors are grateful to K Y Hsieh for performing the TEM measurements. This work was supported by the Ministry of Education and National Science Council of the Republic of China.

\section{References}

[1] Bimberg D, Grundmann M and Ledentsov N N 1999 Quantum Dot Heterostructures (New York: Wiley-Interscience)

[2] Xie Q, Madhukar A, Chen P and Kobayashi N P 1995 Phys. Rev. Lett. 752542

[3] Solomon G S, Trezza J A, Marshall A F and Harris J S Jr 1996 Phys. Rev. Lett. 76952

[4] Heidemeyer H, Kiravittaya S, Müller C, Jin-Phillipp N Y and Schmidt O G 2002 Appl. Phys. Lett. 801544

[5] Lipinski M O, Schuler H, Schmidt O G, Eberl K and Jin-Phillipp N Y 2000 Appl. Phys. Lett. 771789

[6] Santoprete R, Koiller Belita, Capaz R B, Kratzer P, Liu Q K K and Scheffler M 2003 Phys. Rev. B 68235311

[7] Migliorato M A, Wilson L R, Mowbray D J, Skolnick M S, Al-Khafaji M, Cullis A G and Hopkinson M 2001 J. Appl. Phys. 906374

[8] Nuntawong N, Xin Y C, Birudavolu S, Wong P S, Huang S, Hains C P and Huffaker D L 2005 Appl. Phys. Lett. 86193115

[9] Pryor C 1998 Phys. Rev. Lett. 803579

[10] Pryor C 1999 Phys. Rev. B 602869

[11] Toušková J, Samochin E, Toušek J, Oswald J, Hulicius E, Pangrác J, Melichar K and Šimeček T 2002 Appl. Phys. Lett. 9110103

[12] Sun B Q, Lu Z D, Jiang D S, Wu J Q, Xu Z Y, Wang Y Q, Wang J N and Ge W K 1998 Appl. Phys. Lett. 732657

[13] Henini M et al 1998 Phys. Rev. B 57 R6815

[14] Jayavel P, Tanaka H, Kita T, Wada O, Ebe H, Sugawara M, Tatebayashi J, Arakawa Y, Nakata Y and Akiyama T 2004 Appl. Phys. Lett. 841820 